\title{
Object Boundaries Detection Based Center Of Gravity
}

\author{
Ameen A. Noor \\ Department of Computer Science, \\ AL-Mustansiriyh University, \\ College of Education, Baghdad, Iraq
}

\begin{abstract}
After the development in the field of visual data analysis, it has become necessary to obtain objects inside the image, which must be with a high degree of accuracy and clarity for the purpose of identifying the object inside that body in order to obtain clear living organisms, as well as medical professionals can clarify the types of diseases and their forms to find Treatments suitable for her.

In this paper was used a set of previously used and proven mechanisms and techniques that help to remove impurities from the images and reveal the edges to distinguish the object's boundaries and the length of the body and calculate the center of gravity and through the mechanism of the body's rotation to identify these things so that specialists can determine what these things are and how to deal with them And develop possible solutions to it, whether medical, geographical or otherwise.
\end{abstract}

Keywords: Visual Data Analysis, Medical, Center Of Gravity, Body Rotation.

\section{INTRODUCTION}

After the widespread use in the fields of collecting and analyzing visual information related to human activities and geographical locations, and after obtaining this information, which is often captured by video, digital cameras, or even thermal images, or transmitted by means of a communication network, which is often affected by inaccuracies due to video sensors Or dust and dirt on these processing devices, weak light source or angle, or due to transmission through the air, there will be a very clear effect of the inaccuracy of the information captured, which must be reduced by many noise reduction algorithms in addition to the many mechanisms put in place for the signal To the part to be referred to, such as increasing the sharpness of the boundary line of these objects and calculating their lengths to obtain the centers of gravity of these objects, then we carry out the rotation process at an angle that makes it possible to clarify the diseases present within these images for study or the topography and the geographical areas in the image after subjecting them to the mechanisms of detection of boundaries And the edge. Rotation and calculation of the center of gravity of the body to obtain clear and high-resolution images for analyzing the necessary data and procedures on them from solutions.

\section{LITERATURE SURVEY}

The process of visualizing an image inside a computer requires many algorithms that have proven successful in the field of view. The method of Otsu was appropriate in treating computer vision applications by choosing a threshold technique to determine the optimal value of the threshold. It showed its importance [8] as for scanning and identifying the midpoints of the objects (center of gravity). Their work. The edges have proven successful and distinguished [4]. As for the scanning program and determining the average points of objects (center of gravity), it has been experimentally proven to balance objects around a certain point [6].

\section{NOISE REDUCTION}

In order to get rid of the noise inherent in the captured image, which is often formed in more than one way, whether through video, digital camera lens or magnetic resonance, many methods have been used to reduce noise such as (smoothing, 
anisotropic diffusion, given filtering, etc.), the opinion has stabilized. On the use of (anisotropic diffusion) because of its good ability to clarify the image [1], as in

EquationNo.1.

$$
I_{s}^{t+1} \approx I_{s}^{t}+\frac{\lambda}{\left|\eta_{s}\right|} \sum_{p \in \eta_{z}} g\left(\left|\nabla I_{s, p}^{t}\right|, \gamma\right) \nabla I_{s, p}^{t},
$$

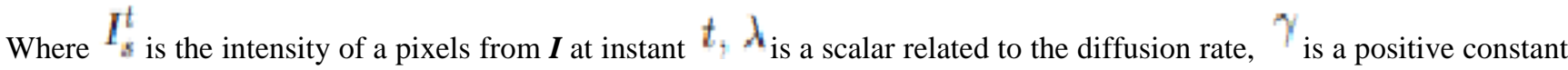
selected according to the desired smoothing level, $\eta_{s}$ stands for the set of adjacent pixelsof s, $g(\cdot)$ is an ESF, and $\nabla I_{s, p}^{t}$ is the magnitude of the image directional gradient from pixel s to $\mathrm{p}$ at instant t.The directional gradiant $\nabla I_{s, p}^{t}$ can be approximated by $I_{p}^{t}-I_{s}^{t}$ To simplify the notation, we will replace $I_{p}^{t}-I_{s}^{t}$ with $x$ whenever pixel information and the iteration number are irrelevant to the context.

\section{OTSU}

In order to reach a high ability to distinguish objects in the field of view within the image, one of the algorithms used in processing binary images must be used. The Otsu method was chosen because it is better in terms of speed and quality, The similarity between two adjacent groups is measured to see the convergence of the two cluster distributions on the graph. When measuring the distance between groups A and B, DistAB uses characteristic analysis adapted from the benchmark function defined by Otsu. The smaller the distance, the better the pair will weld. Therefore, the closest partner is known as the spouse who can be integrated. Since the threshold algorithm can be generalized to deal with a multilevel threshold problem, we can assume that the primary problem is a multilevel threshold problem. The initial values of the parameter $1 \leq \mathrm{k} \leq \mathrm{K}$ can be calculated as follows [2] :

$P_{n}=\operatorname{Pr}\left\{C_{n}\right\}$ is a priori probability of class $n$. Note that $\sum_{k=1}^{\kappa} P_{k}=1$.

$\operatorname{Pr}\left\{z \backslash C_{n}\right\}$ is theprobability density function of gray level $z$ in class $n$. The pixel intensity value $z$ is in the range $[0, L-1]$

The proposed thresholding algorithm is

1. Begin with $\mathrm{K}$ clusters, each consisting of one gray level

- Mean value of cluster $\mathrm{k}, \mathrm{mk}=\mathrm{Tk}$

- $\quad \mathrm{Pk}=\mathrm{h}(\mathrm{Tk})$, where $\mathrm{h}(\mathrm{z})$ is the probability density function of $\mathrm{z}$

2. Repeat step 3-4 a total of $\mathrm{K}-2$ times

3. Compute the distance between adjacent clusters denoted by $\mathrm{k} 1$ and $\mathrm{k} 2$ as follows:

Dist $_{k_{1} k_{2}}=\frac{P_{L_{1}} P_{k_{2}}\left[m_{k_{1}}-m_{k_{2}}\right]^{2}}{\sigma_{k_{1} k_{2}}^{2}}$

Where $\sigma_{i_{1} L_{1}}^{2}$

$M_{k_{1} k_{2}}=\frac{P_{k_{1}} m_{k_{1}}+P_{k_{2}} m_{k_{2}}}{P_{k_{1}}+P_{k_{2}}}$ 
$m_{k}=\sum_{z=T_{i=1}+1}^{T_{i}} z \operatorname{Pr}\left\{z \mid C_{k}\right\}=\frac{1}{P_{k}} \sum_{z=T_{k}+1}^{T_{k}} z h(z)$,

$P_{k}=\operatorname{Pr}\left\{C_{k}\right\}=\sum_{z=T_{k+1}+1}^{T_{k}} h(z)$,

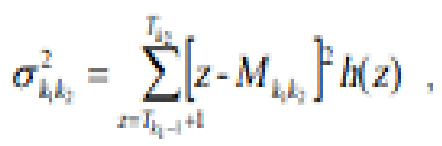

where $\mathrm{M}_{\mathrm{k} 1 \mathrm{k} 2}$ denotes the new mean value of cluster $\mathrm{k} 1$ and $\mathrm{k} 2$, if both clusters are merged.

4. Find the most similar clusters ki and kj, where ii, and remove the last $\mathrm{T}$. At the final step, we will obtain two clusters, with the threshold value denoted by Ti.

\section{EDGE DETECTION}

Edge detection is the first step in covering image information. Borders define the boundaries of objects, so strokes are useful for dividing and defining processes in an image. The purpose of Edge Detection is to increase the appearance of the outlines of an area or object in an image. What the edge perceives is the change in the amount of sudden intensity of (large) gray over a short distance (Wijaya E, 2012)[3-4].

\subsection{Sobel method:}

The Sobel edge detector computes a small slope derivative that can be approximated in a digital image. Typically, grayscale images are used to approximate the absolute slope of any point. Sobel's edge detection technology is based on horizontal and vertical image convolution. The Sobel edge detector is very sensitive to noise[4-5] is as follows:

$$
G=\sqrt{S x^{2}+S y^{2}}
$$

G:Sobel gradient operator value, $S_{x}$ :Horizontal horizontal gradient,

$S_{y}:$ Vertical sobel gradient

\section{CENTER OF GRAVITY}

The center of gravity of the object is calculated according to the given coordinates $(\mathrm{x}, \mathrm{y})$ that belong to the object as shown in the equation:

$$
x_{2 y}=\frac{1}{m} \sum_{i=1}^{m} x_{i}, \quad y_{z \xi}=\frac{1}{m} \sum_{i=1}^{m} y_{i},
$$

where xi, yi are the current coordinates of the contour points along the $\mathrm{X}$ and $\mathrm{Y}$ axes, respectively; $\mathrm{m}$ - number of points object outline[ 6]. 


\section{THINGS ROTATION}

Among the approved algorithms is the eye algorithm used to rotate the object by an angle a, which can calculate the tangent of the angle of the slope of the longitude of the object to the axis through the following formulas[7]:

$$
\begin{aligned}
& x_{\mathrm{H}}=\left(x-x_{\mathrm{L}}\right) \cos \alpha-\left(y-y_{\mathrm{L}}\right) \sin \alpha+x_{\mathrm{L}} ; \\
& y_{\mathrm{H}}=\left(y-y_{\mathrm{L}}\right) \cos \alpha-\left(x-x_{\mathrm{L}}\right) \sin \alpha+y_{\mathrm{L}},
\end{aligned}
$$

where $\mathrm{xH}, \mathrm{yH}$ - new coordinates of the point, $\mathrm{x}, \mathrm{y}$ - original coordinates of the point

\section{PROPOSE METHOD}

The proposed method includes several stages as shown in the figure (1). These steps include : Take the picture And going through several cases to reach the final state, which is the reduction of noise, the use of the Otsu method, the selection of the edge, the calculation of the center of gravity of the body, then the use of the rotation equation.

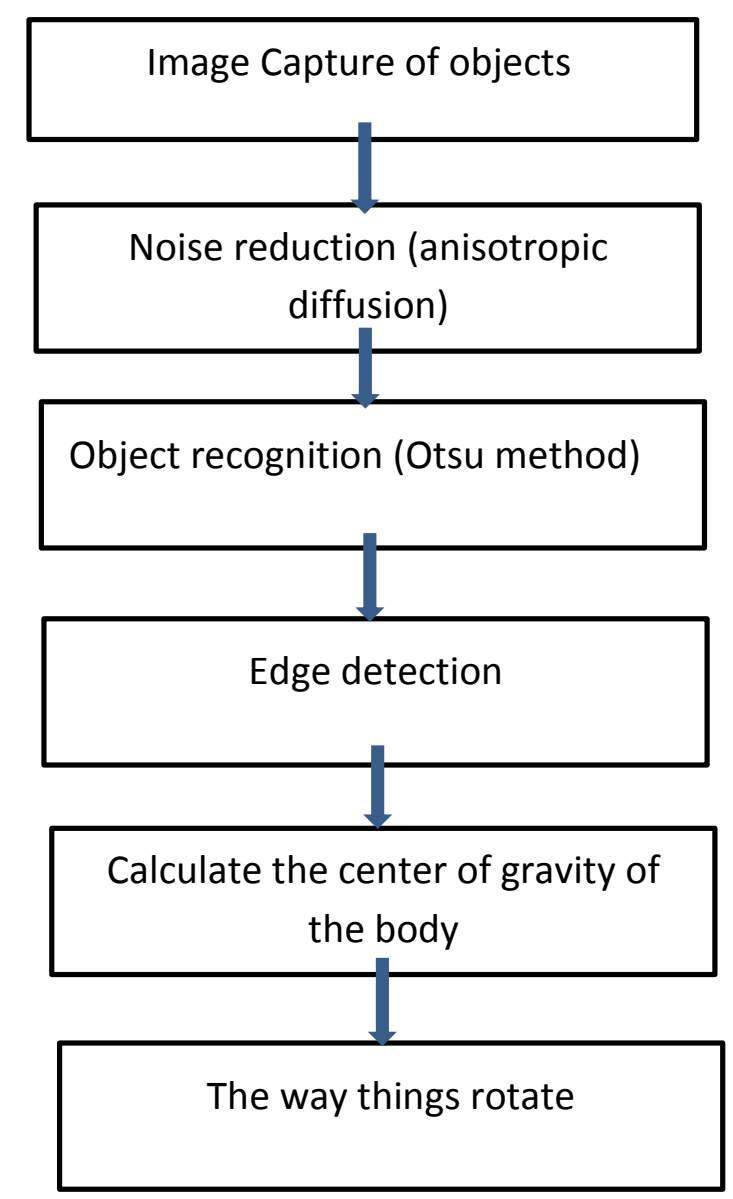

Figure 1. Block diagram of the Proposed Method

\section{RESULTS}

The proposed method was studied, including the studied algorithms, for a set of Images of real flat objects and their preliminary results showed the simplicity of implementation and speed that make the objects within the images can be clarified and distinguished in a high-resolution manner, as shown in the example in the following table. 


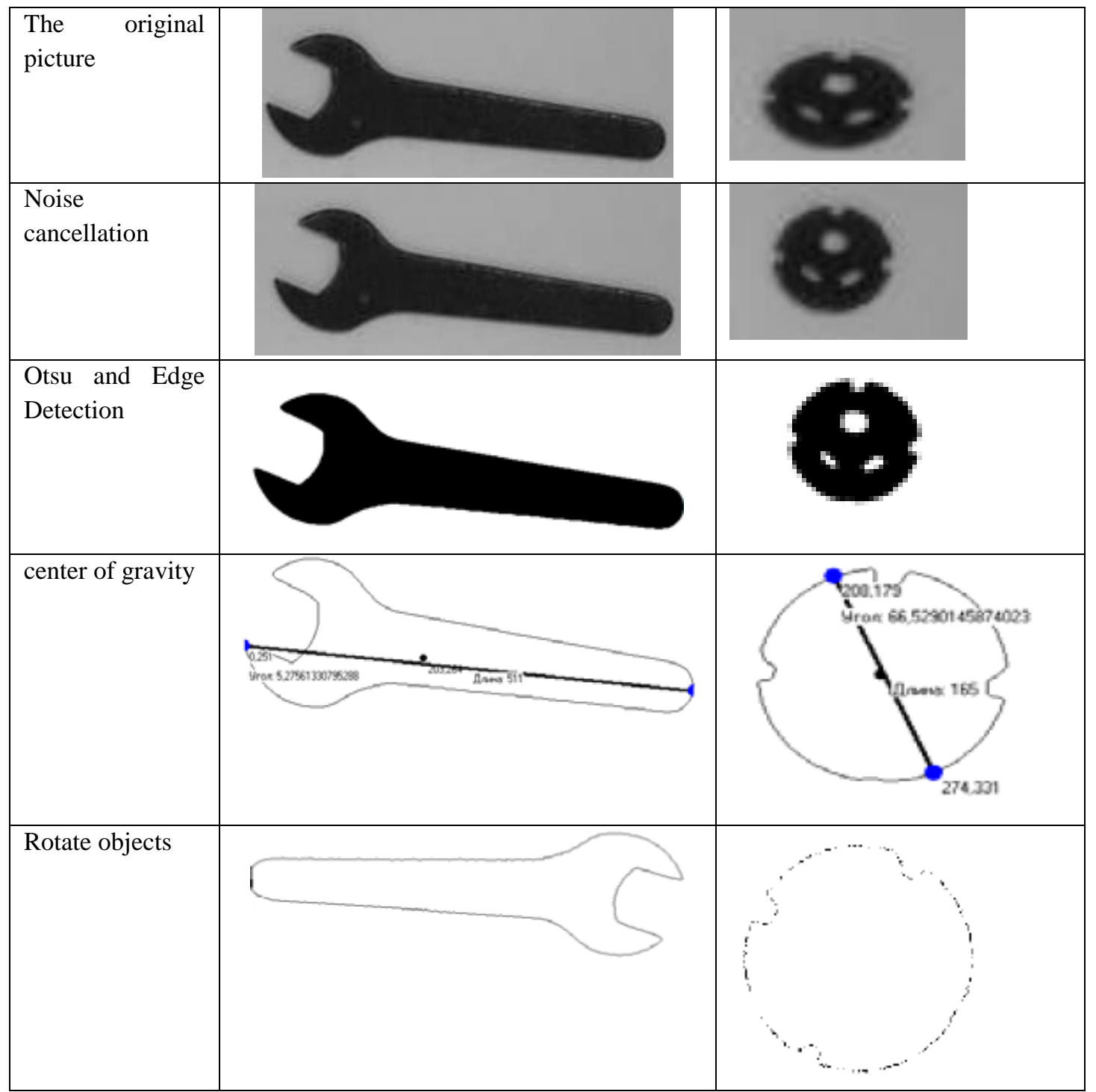

Figure 2: Clarifies the algorithms implemented

\section{CONCLUSIONS}

In this paper, we proposed a set of sequential mechanisms through which impurities are removed from the images and the objects to be studied are determined by strengthening the boundaries of these objects and calculating their lengths and center of gravity and rotating them at an angle through which we can prepare them to be at a high degree of accuracy to take appropriate solutions for these objects and on Different types of it were medical or geographical.

\section{ACKNOWLEDGEMENTS}

The authors would like to thank Mustansiriyah University Baghdad - Iraq (https://uomustansiriyah.edu.iq/) for its support in this work.

\section{REFERENCES}

1- Caio A. Palma, F'abio A.M. Cappabianco, Jaime $S$ " Anisotropic Diffusion Filtering Operation and Limitations Magnetic Resonance Imaging Evaluation" 2014.

2- Agus Zainal Arifin and Akira Asano " Image Thresholding by Histogram

Segmentation Using Discriminant Analysis"2006.

3- Shubhashree Savant" A Review on Edge Detection Techniques for Image Segmentation"2014.

4- Asmaidi Darma Setiawan Putra Muharratul Mina Risky Fitria Ulfa, R" Implementation of Sobel Method Based Edge Detection for Flower Image Segmentation"2019. 
International Journal of Engineering Research And Advanced Technology, Vol.6 (12), December-2020

5- Achal Sharma Shilpa Jaswal " Analysis of Sobel Edge Detection Technique for Face Recognition"2015.

6- Amina Gahramanova "Locating Centers of Mass with Image Processing" 2019

7- Benedict R. Gaster, ... Dana Schaa, in Heterogeneous Computing with OpenCL (Second Edition), 2013.

8- Jamileh Yousefi"Image Binarization using Otsu Thresholding lgorithm" 2018. 